\title{
Diet Quality and Associated Factors Among Eldercare Workers in Long- Term Care Facilities
}

\section{Masao Kanauchi ${ }^{1^{*}}$ and Kimiko Kanauchi ${ }^{2}$}

${ }^{1}$ Department of Health and Nutrition, Faculty of Health Science, Kio University, Koryo-Cho, Japan

${ }^{2}$ Department of Internal Medicine, Narahigashi Hospital, Tenri, Japan

"Corresponding author: Masao Kanauchi, Department of Health and Nutrition, Faculty of Health Science, Kio University, 4-2-2 Umami-Naka, Koryo-Cho Kitakatsuragigun, Nara 635-0832, Japan, Tel: +81745541601; Fax: +81745541600; E-mail: m.kanauchi@kio.ac.jp

Rec Date: Oct 26, 2016, Acc Date: Nov 07, 2016, Pub Date: Nov 09,2016

Copyright: ( $) 2016$ Kanauchi M, et al. This is an open-access article distributed under the terms of the Creative Commons Attribution License, which permits unrestricted use, distribution, and reproduction in any medium, provided the original author and source are credited.

\begin{abstract}
Background: Eldercare workers have a higher prevalence of poor health status, especially in terms of dietary behavior, than general workers. Therefore, the objective of this study was to investigate diet quality and related factors among eldercare workers in long-term care facilities.
\end{abstract}

Methods: We conducted a cross-sectional study on 466 employees in seven long-term care facilities. All participants completed a brief food frequency questionnaire. Diet quality was assessed using three indexes: the American Heart Association healthy diet score (AHA-HDS), the World Health Organization healthy diet index (WHO$\mathrm{HDI}$ ), and the Mediterranean diet score (MDS).

Results: Eldercare workers had significantly lower AHA-HDS and MDS than general workers. Although no differences were seen between the two groups in WHO-HDI scores, a significant positive association was found between all three indexes and combined healthy lifestyle scores (sum of four items: no overweight/obesity, no smoking, adequate alcohol intake, and regular exercise).

Conclusions: This is the first study to report that AHA-HDS and MDS are low in eldercare workers compared with general workers. Assessing dietary quality and improving adherence to healthier dietary patterns in eldercare workers could therefore be expected to aid health promotion.

Keywords: Diet quality; Eldercare; Mediterranean diet

\section{Abbreviations}

AHA-HDS: American Heart Association Healthy Diet Score; BDHQ: Brief Self-administered diet History Questionnaire; BMI: Body Mass Index; DASH: Dietary Approaches to Stop Hypertension; MDS: Mediterranean Diet Score; WHO-HDI: World Health Organization Healthy Diet Index

\section{Introduction}

The eldercare working environment can be burdensome and stressful, especially for elderly residents with dementia [1]. Several studies have reported that adverse working conditions in eldercare, such as stress, a heavy workload, and working in an uncomfortable posture may contribute to an unhealthy lifestyle [2,3]. Job strain has also been linked to unhealthy dietary habits because stress induces food selection changes, uncontrolled eating, intake of high-calorie snacks, and more frequent consumption of fast food [4,5]. It is therefore important to pay more attention to dietary behavior among caregivers and healthcare staff. However, only a few studies have examined dietary behavior in such populations; these studies have reported finding reduced intake of fruits and vegetables [6,7], high-fat diets [6], high intake of sweets [7], and poor dietary diversity [8]. Traditional approaches focusing on specific foods or nutrients were used in these studies, but the findings may be problematic because people consume meals consisting of a variety of foods and complex combinations of nutrients [9]. Recently, increasing comprehensive approaches have emerged to investigate diet quality, resulting in the proposal of several new diet quality indexes and reports of an association between diet quality and health [10]. However, evidence demonstrating an association between diet quality and work settings is limited $[11,12]$, with only one study finding an association between adherence to the dietary approaches to stop hypertension (DASH) diet and obesity among female nurses [13]. To our knowledge, no studies have assessed overall diet quality among eldercare workers. Therefore, the objective of this study was to investigate diet quality and related factors among eldercare workers in long-term care facilities.

\section{Methods}

\section{Participants}

A total of 478 employees at seven long-term care facilities (five nursing homes and two group-living facilities for dementia care) were recruited into this cross-sectional study. After excluding eight participants who had implausibly low or high estimated caloric intake ( $<700$ or $>4500 \mathrm{kcal}$ per day for men and $<600$ or $>3500 \mathrm{kcal}$ per day for women, respectively), and four who had incomplete information, 466 were finally included in the analysis. The participants were then divided into the following two groups: eldercare workers $(n=343 ; 309$ formal caregivers, 28 nurses, and six assistants) and general workers 
( $n=123$; primarily office workers, plus a few kitchen staff, dietitians, janitorial staff, and drivers from the same facilities). The study protocol was approved by the institutional review board of Kio university and written informed consent was obtained from all participants.

\section{Dietary assessments}

Habitual food consumption and nutrient intake were assessed using a brief self-administered diet history questionnaire (BDHQ) [14]. The BDHQ used in this study was a 4-page fixed-portion questionnaire; food and beverage items were selected from foods commonly consumed in Japan, mainly from the food list used in the national health and nutritional survey of Japan [15]. The questionnaire was composed of items on the frequency of consumption of 58 different foods and beverages and required participants to recall their dietary habits over a 1-month period. Participants were asked to indicate how often they had consumed a variety of specific foods over the past month (never, less than once a week, once a week, 2-3 times a week, 4-6 times a week, once-daily, or more than twice a day). Combined with standard serving sizes, the intake frequencies were converted into average daily intake for each food item. Nutrient and energy intake values were then estimated based on questionnaire responses and the corresponding food composition list in the standard tables of food composition in Japan [16].

\section{American Heart Association Healthy Diet Score (AHA- HDS)}

The American Heart Association (AHA) developed five components for a healthy diet score [17]. In this study, the AHA healthy diet score (AHA-HDS) was modified for the Japanese population to include the following five components: $\geq 4.5$ servings of fruits and vegetables per day, $\geq 2$ servings of fish (at least one oily fish) per week, whole grain intake as $\geq 25 \%$ of total grain consumption, $<8 \mathrm{~g}$ of salt intake per day for men and $<7 \mathrm{~g}$ for women (following Japanese dietary reference intake values), and $<1,065 \mathrm{~g}$ of sugar-sweetened beverages per week (equivalent to the original AHA definition of $<36 \mathrm{oz}$ per week). This resulted in scores ranging from $0-5$.

\section{World Health Organization Healthy Diet Index (WHO- HDI)}

The WHO-HDI consists of one food group and eight nutrient components, including fruits and vegetables, carbohydrates, saturated fatty acids, polyunsaturated fatty acids, cholesterol, protein, fiber, free sugar, and sodium [18]. When an intake value was within the range recommended by WHO guidelines [19], a score of 1 was assigned; otherwise, participants received a score of 0 . This resulted in scores ranging from $0-9$.

\section{Mediterranean diet score (MDS)}

According to positions in the updated Mediterranean diet pyramid [20], we constructed an MDS adapted to the Japanese population, as has been described elsewhere [21]. Briefly, the MDS included the following 13 components: fruits, vegetables, grains, legumes, fish, red meat and processed meat, dairy products, potatoes, eggs, poultry, sweets, alcohol, and the ratio of monosaturated to saturated fatty acids. Values of 0 or 1 were assigned for each component, resulting in scores ranging from 0-13.

\section{Other variables}

Body mass index (BMI) was calculated as weight in kilograms divided by the square of height in meters, and overweight/obesity was defined as BMI $\geq 25 \mathrm{~kg} / \mathrm{m}^{2}$. Self-reported questionnaires were used to assess current smoking status (yes, no) and regular exercise ( $\geq 30$ minutes of sweat-inducing exercising at least 2 days a week). Adequate alcohol intake was defined as $10 \mathrm{~g}$ to $30 \mathrm{~g}$ per day for men and $5 \mathrm{~g}$ to 15 $\mathrm{g}$ per day for women. Finally, we developed a combined healthy lifestyle score comprising the following four components: not overweight/obese, no smoking, gets regular exercise, and has adequate alcohol intake. A total score was generated for each item (scored 1 or 0 ), and the sum of the items resulted in a combined healthy lifestyle score (range 0-4).

\section{Statistical Analysis}

Data are presented as means \pm standard deviations or percentages. Differences between groups in mean values of continuous variables were tested using the Students $t$ test, and percentages were compared using chi-square analysis. Differences in nutrient intake, food consumption, and diet quality scores between eldercare and general workers were adjusted for age, and then the age-adjusted means for these dietary variables were compared between groups using analysis of covariance. Linear trends across the graded categories were evaluated with categorical grade as a continuous variable using Spearman's correlation coefficient. Values of $\mathrm{p}<0.05$ were considered statistically significant. All statistical analyses were performed using SPSS (version 21.0; IBM Corp, Armonk, NY).

\section{Results}

The participants' characteristics, nutrient intake, food intake, and scores on the three diet quality indexes are shown in Table 1. Mean age was higher in general workers than in eldercare workers, but BMI was similar between the two groups. Total fat and saturated fatty acid intakes and sweets consumption were significantly lower, and sugarsweetened beverage consumption was significantly higher, in eldercare workers than in general workers. Eldercare workers had significantly lower AHA-HDS and MDS than general workers. On the other hand, no differences were seen in WHO-HDI scores between the two groups.

Table 2 shows the factors related to diet quality in eldercare workers. Men had lower AHA-HDS than women, and younger subjects had lower AHA-HDS and MDS than older subjects. Smokers also had lower AHA-HDS and WHO-HDI scores than nonsmokers. Adequate alcohol intake was related to a higher MDS. A significant positive association was observed between all three diet quality indexes and combined healthy lifestyle scores.

\section{Discussion}

Eldercare workers have a higher prevalence of poor health status, especially in terms of dietary behavior, than general workers [22]. Previous studies have reported that stress is associated with more frequent consumption of sweets and reduced consumption of fruits and vegetables [23]. 
Citation: Kanauchi M, Kanauchi K (2016) Diet Quality and Associated Factors Among Eldercare Workers in Long-Term Care Facilities. J

\begin{tabular}{|c|c|c|c|}
\hline & Eldercare workers & General workers & $\mathbf{p}$ \\
\hline$n$ & 343 & 123 & - \\
\hline Age (years) & $41.2 \pm 12.4$ & $45.9 \pm 12.2$ & $<0.001$ \\
\hline Women (\%) & 72.6 & 75.6 & 0.517 \\
\hline Body mass index $\left(\mathrm{kg} / \mathrm{m}^{2}\right)$ & $22.2 \pm 3.6$ & $22.5 \pm 3.3$ & 0.456 \\
\hline \multicolumn{4}{|l|}{ Nutrient intake } \\
\hline Total energy (kcal/day) & $1845 \pm 647$ & $1778 \pm 655$ & 0.306 * \\
\hline Protein (\% energy) & $14.5 \pm 2.8$ & $14.7 \pm 2.8$ & $0.562 *$ \\
\hline Fat (\% energy) & $25.9 \pm 5.7$ & $27.2 \pm 5.8$ & 0.042 * \\
\hline Carbohydrates (\% energy) & $54.3 \pm 7.8$ & $54.3 \pm 8.8$ & 0.956 * \\
\hline Saturated fatty acids (\% energy) & $7.04 \pm 2.04$ & $7.51 \pm 1.99$ & 0.028 * \\
\hline Salt (g/day) & $12.3 \pm 2.9$ & $12.0 \pm 3.0$ & 0.350 * \\
\hline \multicolumn{4}{|l|}{ Food consumption } \\
\hline Grains (g/day) & $481 \pm 178$ & $471 \pm 180$ & 0.589 * \\
\hline Vegetables (g/day) & $256 \pm 135$ & $277 \pm 135$ & 0.143 * \\
\hline Fruits (g/day) & $115 \pm 120$ & $100 \pm 121$ & 0.223 * \\
\hline Meat (g/day) & $85 \pm 42$ & $89 \pm 43$ & 0.443 * \\
\hline Fish (g/day) & $85 \pm 50$ & $81 \pm 49$ & $0.447^{*}$ \\
\hline Dairy products (g/day) & $142 \pm 124$ & $152 \pm 124$ & 0.453 * \\
\hline Sweets (g/day) & $54 \pm 42$ & $64 \pm 43$ & 0.032 * \\
\hline Sugar-sweetened beverage (g/day) & $129 \pm 177$ & $77 \pm 179$ & 0.006 * \\
\hline \multicolumn{4}{|l|}{ Diet quality indexes } \\
\hline AHA-HDS & $1.97 \pm 0.92$ & $2.22 \pm 1.00$ & 0.019 * \\
\hline WHO-HDI & $4.07 \pm 1.11$ & $3.97 \pm 1.22$ & 0.393 * \\
\hline MDS & $5.08 \pm 1.67$ & $5.42 \pm 1.55$ & 0.044 * \\
\hline
\end{tabular}

Table 1: Participants' characteristics, nutrient intake, food intake, and scores on the three diet quality indexes used in this study.

In the present study, the consumption of sugar-sweetened beverages was higher among eldercare workers than general workers, but no differences were seen between groups in the consumption of fruits and vegetables. Conversely, although previous studies reported that individuals ingest more fat following exposure to stress [4,5], in the present study, we found that eldercare workers consumed significantly lower amounts of sweets, total fat, and saturated fatty acids than general workers. These findings might be explained by the fact that eldercare workers experience both physical fatigue and mental stress, and these conditions may affect dietary behavior, such as promoting a preference for lighter foods or sugar-sweetened drinks, or an avoidance of greasy/oily foods such as Western-style confectioneries or high-fat snacks.

Recently, the use of diet quality index has emerged as an alternative to single food- or nutrient-based approaches to examine associations between dietary behavior and health [10]. The primary strength of our study was the use of comprehensive measures of diet quality that can provide a more accurate picture of overall dietary habits. This approach allows information regarding food and nutrient consumption to be combined into a simple numerical score. 
Page 4 of 5

\begin{tabular}{|c|c|c|c|c|c|c|c|c|}
\hline Factor & & $\mathrm{n}$ & AHA-HDS & $\mathbf{p}$ & WHO-HDI & $\mathbf{p}$ & MDS & $\mathbf{p}$ \\
\hline \multirow[t]{2}{*}{ Sex } & Male & 94 & $1.73 \pm 1.03$ & \multirow[t]{2}{*}{0.023} & $4.18 \pm 1.02$ & \multirow[t]{2}{*}{0.275} & $5.29 \pm 1.69$ & \multirow[t]{2}{*}{0.106} \\
\hline & Female & 249 & $2.02 \pm 1.03$ & & $4.03 \pm 1.20$ & & $4.97 \pm 1.59$ & \\
\hline \multirow[t]{2}{*}{ Age (years) } & $\geq 50$ & 93 & $2.31 \pm 0.86$ & \multirow[t]{2}{*}{0.001} & $4.15 \pm 1.14$ & \multirow[t]{2}{*}{0.432} & $5.39 \pm 1.55$ & \multirow[t]{2}{*}{0.021} \\
\hline & $<50$ & 250 & $1.80 \pm 1.07$ & & $4.04 \pm 1.16$ & & $4.93 \pm 1.65$ & \\
\hline \multirow[t]{2}{*}{ Obesity } & Yes & 69 & $1.88 \pm 0.92$ & \multirow[t]{2}{*}{0.607} & $3.94 \pm 1.26$ & \multirow[t]{2}{*}{0.304} & $4.75 \pm 1.50$ & \multirow[t]{2}{*}{0.085} \\
\hline & No & 274 & $1.96 \pm 1.07$ & & $4.10 \pm 1.13$ & & $5.13 \pm 1.65$ & \\
\hline \multirow[t]{2}{*}{ Smoking } & Yes & 106 & $1.63 \pm 0.99$ & \multirow[t]{2}{*}{0.001} & $3.83 \pm 1.02$ & \multirow[t]{2}{*}{0.010} & $5.05 \pm 1.63$ & \multirow[t]{2}{*}{0.950} \\
\hline & No & 237 & $2.08 \pm 1.03$ & & $4.18 \pm 1.20$ & & $5.06 \pm 1.63$ & \\
\hline \multirow{2}{*}{$\begin{array}{l}\text { Adequate alcohol } \\
\text { intake }\end{array}$} & Yes & 31 & $2.00 \pm 1.21$ & \multirow[t]{2}{*}{0.744} & $4.32 \pm 1.25$ & \multirow[t]{2}{*}{0.202} & $5.74 \pm 1.63$ & \multirow[t]{2}{*}{0.014} \\
\hline & No & 312 & $1.94 \pm 1.02$ & & $4.04 \pm 1.14$ & & $4.99 \pm 1.62$ & \\
\hline \multirow[t]{2}{*}{ Regular exercise } & Yes & 127 & $1.96 \pm 1.04$ & \multirow[t]{2}{*}{0.856} & $4.11 \pm 1.18$ & \multirow[t]{2}{*}{0.648} & $5.06 \pm 1.68$ & \multirow[t]{2}{*}{0.948} \\
\hline & No & 215 & $1.94 \pm 1.03$ & & $4.05 \pm 1.14$ & & $5.05 \pm 1.61$ & \\
\hline \multirow[t]{3}{*}{ CHLS } & $0-1$ & 99 & $1.76 \pm 1.83$ & \multirow[t]{3}{*}{$0.001^{*}$} & $3.90 \pm 2.13$ & \multirow[t]{3}{*}{$0.034^{*}$} & $4.95 \pm 2.99$ & \multirow[t]{3}{*}{$0.010^{*}$} \\
\hline & 2 & 160 & $1.99 \pm 1.44$ & & $4.01 \pm 1.68$ & & $5.00 \pm 2.35$ & \\
\hline & $3-4$ & 84 & $2.06 \pm 1.99$ & & $4.38 \pm 2.31$ & & $5.29 \pm 3.26$ & \\
\hline
\end{tabular}

Table 2: Factors related to diet quality in eldercare workers.

We used three indexes to assess diet quality in this study. The first, the AHA-HDS is one component of the AHA's seven metrics for ideal cardiovascular health [17]. It was developed with the aim of reducing cardiovascular disease mortality and improving cardiovascular health by $20 \%$ by the year 2020 and beyond [17]. With only five components, this index is very simple and easy to apply in clinical practice. To the best of our knowledge, no previous studies have compared adherence to the AHA-HDS between eldercare and general workers. In the present study, we found that eldercare workers had lower AHA-HDS than general workers. Indeed, it has been recognized that dietary patterns differ by nationality, and traditional Japanese dietary habits differ from those in Western populations. However, the contemporary diet of most Japanese adults has come to resemble Western diets. Therefore, we believe that it is reasonable to assess AHA-HDS in the Japanese population.

The second index, the WHO-HDI, is a tool developed to assess compliance with the WHO's dietary guidelines for the prevention of chronic disease [18]. This index has been used worldwide and studied in relation to all-cause mortality, overall cancer risk, and cardiovascular disease risk [24]; however, no studies have investigated the association between this index and the dietary behaviors of caregivers. In the present study, no differences were seen in WHO-HDI scores between eldercare and general workers. Although the AHAHDS is based mostly on food groups, the WHO-HDI is primarily based on nutrients, except for the component for consumption of fruits and vegetables. This indicates that that dietary behavior among eldercare workers might be more strongly associated with diet quality based on food groups.
The third index, the MDS, is widely known for its use in assessing the habitual intake of a Mediterranean-style diet. It could be said that Japanese people have not adopted a Mediterranean-style diet, and little is known about habitual adherence to such a diet in Japan [25]. However, it is well documented that Japanese people traditionally eat high quantities of fish, vegetables, and legumes, and low quantities of meat. Some components of this dietary pattern are similar to that of a Mediterranean-style diet. We therefore speculate that a considerable number of Japanese people might adhere to a Mediterranean-style diet. We demonstrated that Japanese eldercare workers had significantly lower MDS than general workers. A Mediterranean-style diet includes high levels of antioxidants and anti-inflammatory compounds, and its beneficial effects include a reduction of risk for metabolic and cardiovascular diseases. There is little doubt that the habitual intake of a Mediterranean-style diet provides significant health benefits [26]. This suggests that eldercare workers with low adherence to a Mediterranean-style diet should be encouraged to adopt healthier dietary behaviors.

In the present study, all diet quality indexes were significantly associated with a combined healthy lifestyle score. This means that eldercare workers with an adherence to high quality diet may have a healthy lifestyle. There has been a growing interest in the combined impact of habitual healthy behaviors on overall health [27]. Previous studies also reported an association between diet quality and a clustering of healthy lifestyles [28,29]. But, causality between diet quality and other healthy behaviors cannot be drawn from the present study. Further study is needed to determine the association between 
the combined lifestyle factors including diet quality and health outcomes in eldercare workers.

This study did have several limitations. First, because this was a cross-sectional study, the analysis cannot prove a causal relationship between the dietary quality indexes and the healthy lifestyles of eldercare workers. Second, because our results only showed associations among workers at a small number of long-term care facilities, our findings may not be generalizable to other care populations in different work areas. Third, chronic stress at work is known to be associated with unhealthy dietary habits, but the present study did not evaluate the degree of caregiver burden. Finally, olive oil is an important component of a Mediterranean-style diet, but Japanese people are known to consume relatively low amounts of olive oil. In addition, the BDHQ used in the present study did not estimate olive oil intake. As opposed to olive oil consumption, we used an indirect parameter, the monounsaturated/saturated fatty acid ratio, which is frequently used in the traditional MDS.

In conclusion, eldercare workers had an overall low diet quality, especially according to the AHA-HDS and MDS. Eldercare workers with low diet quality also had unhealthy lifestyles. Assessing dietary quality and improving adherence to healthier dietary patterns could therefore be expected to aid health promotion among eldercare workers.

\section{Author Contribution}

MK designed the study, researched the data and wrote the manuscript. KK contributed to the data collection and the discussion. All authors approved the final version for submission.

\section{References}

1. Jakobsen LM, Jorgensen AFB, Thomsen BL, Greiner BA, Rugulies R (2015) A multilevel study on the association of observer-assessed working conditions with depressive symptoms among female eldercare workers from 56 work units in 10 care homes in Denmark. BMJ Open 5: e008713.

2. Pitfield C, Shahriyarmolki K, Livingston G (2011) A systematic review of stress in staff caring for people with dementia living in 24-hour care settings. Int Psychogeriatr 23: 4-9.

3. de Rooij AH, Luijkx KG, Declercq AG, Emmerink PM, Schols JM (2012) Professional caregivers' mental health problems and burnout in smallscale and traditional long term care settings for elderly people with dementia in the Netherlands and Belgium. J Am Med Dir Assoc 13: 486.

4. Richardson AS, Arsenault JE, Cates SC, Muth MK (2015) Perceived stress, unhealthy eating behaviors, and severe obesity in low-income women. Nutr J 14: 122.

5. Yau YH, Potenza MN (2013) Stress and eating behaviors. Minerva Endocrinol 38: 255-267.

6. Zapka JM, Lemon SC, Magner RP, Hale J (2009) Lifestyle behaviors and weight among hospital-based nurses. J Nurs Manag 17: 853-860.

7. Mota MC, De-Souza DA, Rossato LT, Silva CM, Araujo MB, et al. (2013) Dietary patterns, metabolic markers and subjective sleep measures in resident physicians. Chronobiol Int 30: 1032-1041.

8. Oldewage-Theron W, Kruger R (2011) Dietary diversity and adequacy of women caregivers in a peri-urban informal settlement in South Africa. Nutrition 27: 420-427.

9. Gerber M (2001) The comprehensive approach to diet. A critical review. J Nutr 131: 3051S -3055S.

10. Kourlaba G, Panagiotakos DB (2009) Dietary quality indices and human health: A review. Maturitas 62: 1-8.
11. Previdelli AN, Lipi M, Castro MA, Marchioni DM (2010) Dietary quality and associated factors among factory workers in the metropolitan region of Sao Paulo, Brazil. J Am Diet Assoc 110: 786-790.

12. Kuroki Y, Kanauchi K, Kanauchi M (2012) Adherence index to the American Heart Association Diet and Lifestyle Recommendation is associated with the metabolic syndrome in Japanese male workers. Eur J Intern Med 23: 199-203.

13. Barak F, Falahi E, Keshteli AH, Yazdannik A, Esmaillzadeh A (2015) Adherence to the Dietary Approaches to Stop Hypertension (DASH) diet in relation to obesity among Iranian female nurses. Public Health Nutr 18: 705-712.

14. Kobayashi S, Murakami K, Sasaki S, Okubo H, Hirota N, et al. (2011) Comparison of relative validity of food group intakes estimated by comprehensive and brief-type self-administered diet history questionnaires against $16 \mathrm{~d}$ dietary records in Japanese adults. Public Health Nutr 11: 1-12.

15. Ministry of Health, Labour, and Welfare of Japan (2008) The National Health and Nutrition Survey in Japan. Ministry of Health, Labour, and Welfare.

16. http://www.fao.org/infoods/infoods/tables-and-databases/japan/en/

17. Lloyd-Jones DM, Hong Y, Labarthe D, Mozaffarian D, Appel LJ, et al. (2010) Defining and setting national goals for cardiovascular health promotion and disease reduction: the American Heart Association's strategic Impact Goal through 2020 and beyond. Circulation 121: 586-613.

18. Huijbregts P, Feskens E, Rasanen L, Fidanza F, Nissinen A, et al. (1997) Dietary pattern and 20 years mortality in elderly men in Finland, Italy, and the Netherlands. BMJ 315: 13-17.

19. WHO (2003) Diet, nutrition, and prevention of chronic diseases. Report of a WHO Study Group. WHO Tech Rep Ser 916: 1-149.

20. Bach-Faig A, Berry EM, Lairon D, Reguant J, Trichopoulou A, et al. (2011) Mediterranean diet pyramid today. Public Health Nutr 14: 2274-2284.

21. Kanauchi M, Kanauchi K (2016) Development of a Mediterranean diet score adapted to Japan and its relation to obesity risk. Food Nutr Res 60: 32172.

22. Miranda H, Gore RJ, Boyer J, Nobrega S, Punnett L (2015) Health behaviors and overweight in nursing home employees. Sci World J : 915359.

23. Mikolajczyk RT, El Ansari W, Maxwell AE (2009) Food consumption frequency and perceived stress and depressive symptoms among students in three European countries. Nutr J 8: 31.

24. Jankovic N, GeelenA, Streppel MT, de Groot L, Kiefte-de Jong JC, et al. (2015) WHO guidelines for a healthy diet and mortality from cardiovascular disease in European and American elderly. Am J Clin Nutr 102: 745-756.

25. Kanauchi M, Kanauchi K (2015) Diet quality and adherence to a healthy diet in Japanese male workers with untreated hypertension. BMJ Open 5: e008404.

26. Grosso G, Mistretta A, Frigiola A, Gruttadauria S, Biondi A, et al. (2014) Mediterranean diet and cardiovascular risk factors: a systematic review. Crit Rev Food Sci Nutr 54: 593-610.

27. Petersen KE, Johnsen NF, Olsen A, Albieri V, Olsen LK, et al. (2015) The combined impact of adherence to five lifestyle factors on all-cause, cancer and cardiovascular mortality. Br J Nutr 113: 849-858.

28. Patino-Alonso MC, Recio-Rodriguez JI, Belio JFM, Colominas-Garrido R, Lema-Bartolome J, et al. (2014) Factors associated with adherence to the Mediterranean diet in the adult population. J Acad Nutr Diet 114: 583-589.

29. Roswall N, Eriksson U, Sandin S, Lof M, Olsen A, et al. (2015) Adherence to the healthy Nordic food index, dietary composition, and lifestyle among Swedish women. Food Nutr Res 59: 26336. 